\title{
Evidence for a promoter of urate crystal formation in gouty synovial fluid
}

Rheumatology Unit, Bristol Royal Infirmary, Bristol

N W McGill

P A Dieppe

Correspondence to: Dr N W McGill Rachel Forster Hospital, 150 Pitt Street, Redfern, NSW 2016, Australia.

Accepted for publication 1 August 1990

\begin{abstract}
The effect of serum and synovial fluid obtained from six healthy subjects and from 12 patients with gout, six with rheumatoid arthritis and 18 with calcium pyrophosphate dihydrate arthropathy (CPPD) on the rate of in vitro urate crystal formation was measured. Gouty and normal serum produced similar results. Gouty synovial fluids promoted urate crystal formation significantly more than did rheumatoid or CPPD fluids. The promotion of urate crystal formation by gouty synovial fluid was not due to native urate crystals nor to an effect on the level of urate supersaturation. The development of gout in some, but not other, hyperuricaemic subjects may relate to the presence of promoters (or relative lack of inhibitors) of urate crystal formation in the group who develop gout.
\end{abstract}

Although hyperuricaemia is necessary for the development of gout, only a minority of hyperuricaemic subjects ever develop gout. ${ }^{1}$ Furthermore, the correlation between the development of gout and the level of hyperuricaemia, although definitely present, is poor and it seems that only a subgroup of hyperuricaemic subjects is at risk for the development of gout. ${ }^{1}$ It thus seems likely that factors other than hyperuricaemia influence the development of gout.

The formation of crystals of monosodium urate monohydrate (urate) is an essential and probably critical step between asymptomatic hyperuricaemia and gout. Crystal formation is dependent on the effective level of supersaturation of the component ions of the crystal in the surrounding milieu (in this case the [sodium] and [urate] activities) but is also influenced by the potential presence of substances which affect nucleation or growth rate. It has been suggested ${ }^{2-5}$ that such substances may play a significant part in determining susceptibility to gout. In vivo, urate crystals are predominantly found in the superficial layer of synovium and articular cartilage and in synovial fluid. It is logical, therefore, to expect that if promoters/inhibitors of urate nucleation or growth are active they should be present in synovial fluid, synovium, or cartilage.

Three small studies on the effect of hyperuricaemia on urate crystal formation have suggested that gouty synovial fluid may contain a promoter of urate crystal nucleation, though the numbers of fluids tested were too few to allow statistical significance testing. ${ }^{2} 6$ No study on the effect of serum or plasma from gouty patients on urate crystal formation has been published, though the effect of normal serum has recently been reported. ${ }^{7}$ a

We examined the effects of serum samples and synovial fluids from gouty patients on in vitro urate crystal formation. Serum samples and synovial fluids from normal subjects and patients with calcium pyrophosphate dihydrate arthropathy (CPPD) or rheumatoid arthritis served as controls.

\section{Patients and methods}

The study was approved by the local ethical committee.

\section{PATIENTS AND CONTROLS}

All gouty patients included in the study had a characteristic history and had had urate crystals identified in synovial fluid. Patients with rheumatoid arthritis all had four or more American Rheumatism Association criteria. ${ }^{8}$ The CPPD control group comprised patients with clinical osteoarthritis who were selected because of the presence of calcium pyrophosphate dihydrate crystals in their synovial fluid aspirates. All had chronic symptoms and none of the fluids was obtained during an attack of pseudogout. Departmental members served as healthy controls-none of whom had a history of gout, rheumatoid arthritis, or osteoarthritis.

\section{PREPARATION OF SAMPLES}

Blood was collected into plain plastic tubes, allowed to clot, centrifuged gently $(1000 \mathrm{rpm}$, five minutes), and the serum removed. Serum was then stored at $-70^{\circ} \mathrm{C}$ until used. Synovial fluid was aspirated and promptly examined by plain and polarised light microscopy for the presence of crystals. All gouty synovial fluids contained urate crystals and all CPPD synovial fluids contained crystals typical of calcium pyrophosphate dihydrate. Synovial fluids were centrifuged hard (4100 rpm, 15 minutes) and the supernatant stored at $-70^{\circ} \mathrm{C}$ until used. Serum and synovial fluid supernatants were thawed and warmed to $37^{\circ} \mathrm{C}$ before use. An aliquot of each synovial fluid supernatant was reserved for polarised light and electron microscopy.

All studies were performed using paired samples. For the serum studies each gouty sample was paired with a healthy control sample (six pairs). In the synovial fluid studies the pairings were gout-CPPD (12 pairs) or rheumatoid arthritis-CPPD (six pairs). 
URATE CRYSTAL FORMATION

The effect of each pair of samples of serum or synovial fluid upon the rate of urate crystal formation from a highly supersaturated solution of sodium urate at $\mathrm{pH} 7 \cdot 4,37^{\circ} \mathrm{C}$ was measured as previously described. ${ }^{7} 7 \mathrm{a}$ Briefly, an equimolar supersaturated solution of sodium urate was prepared by adding uric acid to a hot solution of sodium hydroxide, after which the $\mathrm{pH}$ was adjusted with sodium hydroxide to $7 \cdot 4$ and the solution allowed to cool to $37^{\circ} \mathrm{C}$. The solution was filtered ( $0.22 \mu \mathrm{m}$ Millipore filters) before use and all glassware was thoroughly cleaned. Glass distilled water was used throughout and special measures were taken to avoid dust contamination. Aliquots $(5 \mathrm{ml})$ of the supersaturated urate solution were mixed with $0.5 \mathrm{ml}$ aliquots of serum or synovial fluid diluted in phosphate buffered saline such that the final concentration of serum or synovial fluid in the crystal growth jars varied between $9 \%$ and $0 \%$ (no serum/synovial fluid added).

The crystal growth jars were then incubated at $37^{\circ} \mathrm{C}$ for four or eight hours, after which the contents were filtered through preweighed Millipore filters to remove the crystals. The filtrates were stored at $-70^{\circ} \mathrm{C}$. The filters were dried at $37^{\circ} \mathrm{C}$ and reweighed, thus allowing the weight of the formed crystals to be determined. Controls (zero incubation period) were similarly treated in each experiment to adjust for the weight of added serum or synovial fluid. Filters $(0.22 \mu \mathrm{m})$ were used for the serum experiments and $5.0 \mu \mathrm{m}$ filters for the synovial fluid experiments. Attempts to use filters with a smaller pore size for synovial fluid were unsuccessful owing to blocking of the filter. Potential loss of crystal through the $5.0 \mu \mathrm{m}$ filters was assessed by filtering suspensions of urate crystals in water or serum through $5.0 \mu \mathrm{m}$ filters and then passing the filtrate through $0.22 \mu \mathrm{m}$ filters. In all cases the loss of crystal through the $5.0 \mu \mathrm{m}$ filter was less than the detection threshold of the method (representing a maximum error of about $1 \%)$. The urate concentration of each filtrate from one set of experiments (9\% synovial fluid, eight hour incubation) was subsequently determined with a commercial multiple biochemical analyser which relies on a colorimetric method for determining urate concentration.

STATISTICS

Analyses were performed with the Wilcoxon matched pairs test.

Results

All figures are expressed as mean (standard deviation).

\section{SUBJECTS}

The table gives demographic details of the subjects taking part in each section of the study. The age in years of each group was $(a)$ healthy $v$ gout serum study: healthy $34 \cdot 2(5 \cdot 3)$, gout $46 \cdot 3$ $(8 \cdot 1) ;(b)$ rheumatoid arthritis $v$ gout synovial
Age and sex of the subjects taking part in the three sections of the study

\begin{tabular}{|c|c|c|c|c|c|}
\hline \multicolumn{2}{|c|}{ Serum studies } & \multicolumn{4}{|c|}{ Synovial fluid studies } \\
\hline Healthy & Gout & $R A^{*}$ & Gout & $R A$ & $C P P D^{*}$ \\
\hline $\begin{array}{l}29 M \\
31 M \\
40 M \\
32 M \\
30 M \\
43 M\end{array}$ & $\begin{array}{l}56 M \\
50 M \\
44 M \\
31 M \\
44 M \\
53 M\end{array}$ & $\begin{array}{l}75 \mathrm{~F} \\
63 \mathrm{~F} \\
79 \mathrm{~F} \\
74 \mathrm{~F} \\
35 \mathrm{~F} \\
75 \mathrm{~F} \\
62 \mathrm{M} \\
59 \mathrm{M} \\
66 \mathrm{~F} \\
65 \mathrm{M} \\
66 \mathrm{~F} \\
52 \mathrm{M}\end{array}$ & $\begin{array}{l}43 M \\
68 F \\
51 M \\
36 M \\
33 M \\
69 M \\
76 M \\
53 M \\
55 M \\
63 M \\
50 M \\
63 M\end{array}$ & $\begin{array}{l}52 M \\
66 F \\
74 F \\
35 F \\
62 M \\
75 F\end{array}$ & $\begin{array}{l}73 M \\
65 M \\
64 M \\
77 F \\
82 F \\
77 F\end{array}$ \\
\hline
\end{tabular}

${ }^{*} \mathrm{RA}=$ rheumatoid arthritis; $\mathrm{CPPD}=$ calcium pyrophosphate dihydrate arthropathy.

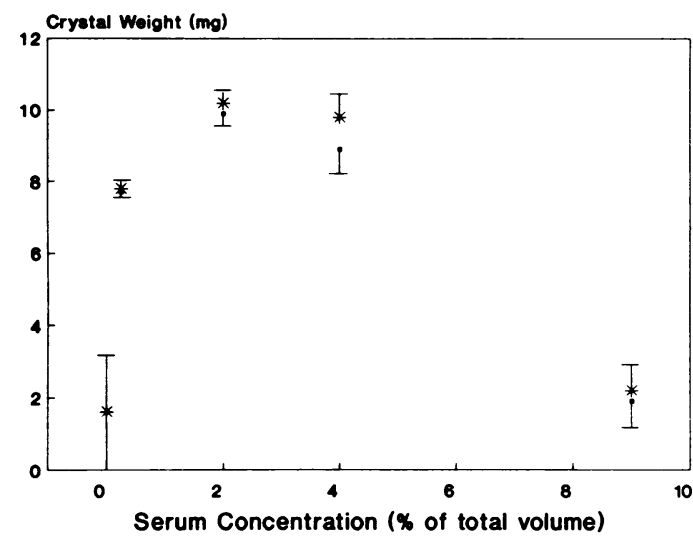

Figure 1 Urate crystal formation in the presence of gouty $\left({ }^{*}\right)$ or normal (-) serum. $0 \cdot 25 \%, 2 \%$, and $4 \%$ serum all significantly promoted urate crystal formation compared with control $(p<0 \cdot 001)$. Gouty and normal serum were not significantly different.

fluid study: rheumatoid arthritis $64 \cdot 3(11 \cdot 5)$, gout $55.0(12 \cdot 8)$; (c) rheumatoid arthritis $v$ CPPD synovial fluid study: rheumatoid arthritis 60.7 (13.8), CPPD $73(6 \cdot 6)$.

SERUM

Figure 1 shows the effect of normal and gouty serum on urate crystal formation. The results for normal serum confirmed our previous findings ${ }^{7 \mathrm{7a}}$ of a concentration dependent promotion of urate crystal formation, most marked at low $(2-4 \%)$ concentrations. No difference between the effect of normal and gouty serum was found.

SYNOVIAL FLUID

All synovial fluid samples (from subjects with gout, rheumatoid arthritis, or CPPD) produced a change in the rate of urate crystal formation which was qualitatively similar to that produced by serum. When the concentration of synovial fluid in the crystal growth jar was low (2\%) a significant $(\mathrm{p}<0.001)$ promotion of urate crystal formation occurred, whereas at higher concentration (9\%) this effect was markedly reduced. Gouty synovial fluid promoted urate crystal formation significantly more than did rheumatoid synovial fluid, both at $2 \%(\mathrm{p}<0.001)$ and $9 \%(p<0.01)$ concentration (fig 2$)$. In a typical set of experiments, after four hours' incubation, 


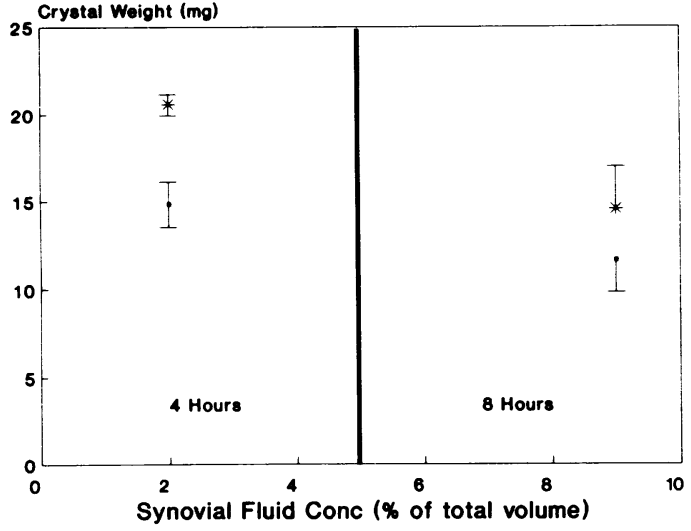

Figure 2 Urate crystal formation in the presence of gouty ${ }^{*}$ ) or rheumatoid $(\bullet)$ synovial fluid. Left: four hour incubation, $2 \%$ synovial fluid; $p<0.001$. Right: eight hour incubation, $9 \%$ synovial fluid; $p<0 \cdot 01$.

urate crystal formation in each crystal growth jar was $20.6(0.6) \mathrm{mg}$ in the presence of $2 \%$ gouty synovial fluid and $14.8(1.3) \mathrm{mg}$ in the presence of $2 \%$ rheumatoid synovial fluid, corresponding to a $39 \%$ excess of crystal formation in the presence of gouty synovial fluid. After eight hours' incubation urate crystal formation in each crystal growth jar was 14.5 $(2.5) \mathrm{mg}$ in the presence of $9 \%$ gouty synovial fluid and $11.6(1.8) \mathrm{mg}$ in the presence of $9 \%$ rheumatoid synovial fluid, corresponding to a $25 \%$ excess of crystal formation in the presence of gouty synovial fluid.

The urate concentration in the filtrate was significantly lower when crystal formation had occurred in the presence of gouty $(14 \cdot 1(3 \cdot 2)$ $\mathrm{mmol} / \mathrm{l})$ compared with rheumatoid $(16 \cdot 4(3 \cdot 3)$ $\mathrm{mmol} / \mathrm{l})$ synovial fluid $(\mathrm{p}<0.05)$, indicating that the excess promotion of crystal formation in the presence of gouty synovial fluid was not simply due to an increase in the level of urate supersaturation.

To explore the possibility that the excess promotion of urate crystal formation found in the presence of gouty synovial fluid was due to the presence of native urate crystals (which centrifugation had failed to remove) in these fluids we examined an aliquot of each gouty synovial fluid supernatant by polarised light and transmission electron microscopy and energy dispersive $x$ ray analysis. No urate crystal was identified in any supernatant.

Rheumatoid and CPPD synovial fluids had almost identical effects on the rate of urate crystal formation, with no significant difference between the two groups.

\section{Discussion}

This study showed that gouty synovial fluid promotes urate crystal formation through an effect independent of the level of urate supersaturation. Urate crystal formation is the key step between asymptomatic hyperuricaemia and gout. As with crystal formation generally, it requires a sufficient sodium urate activity product (the medium in which the crystal forms must be supersaturated with respect to sodium urate) but, in addition, it is influenced by the presence of factors that affect nucleation and crystal growth. That these factors are important in vivo is clearly shown by the restricted distribution of urate crystal deposition (joints and other connective tissues) in gouty patients despite a similar level of urate supersaturation throughout the body. Although studies on the effect of individual tissue components on urate crystal formation have been reported, ${ }^{2-69-12}$ the findings have been conflicting, and the factors which determine the distribution of urate deposition within the body remain unclear.

Epidemiological studies have suggested that only a subgroup of hyperuricaemic subjects are at risk for gout, ${ }^{1}$ but no risk factor for gout that does not act through hyperuricaemia has yet been identified. ${ }^{13}$ It seems likely, however, that the reason some hyperuricaemic subjects never develop gout and other equally hyperuricaemic subjects do, is related to a difference in the presence or concentration of crystal nucleators or growth inhibitors, or both. Previous studies of the effect of synovial fluid on urate crystal formation have been limited. Khalaf and Wilcox reported that synovial fluid (at $5 \%$ concentration) from one gouty patient enhanced, and from two rheumatoid patients inhibited, urate crystal formation, an effect which they felt was due to an alteration in the rate of nucleation. ${ }^{6}$ The same authors subsequently reported the same findings with synovial fluid obtained from one gouty and one rheumatoid patient, ${ }^{2}$ though it is not clear whether this latter report derived from the same data or not. Tak, Cooper, and Wilcox reported 'marked' enhancement of urate crystal formation in the presence of four gouty synovial fluids, 'moderate' enhancement in the presence of three osteoarthritic fluids, and only a 'slight effect' from five rheumatoid fluids. ${ }^{3}$ In their study the effect of synovial fluid was measured at only one concentration $(10 \%)$ and one time point. Although it was stated that urate crystals were not in the gouty fluids before use, no detail of how this was determined was given. The method used allowed only qualitative results and no statistical analysis was provided, presumably because of the small numbers studied.

No previous study of the effect of gouty serum on the rate of urate crystal formation has been reported. Although serum has a pronounced concentration dependent effect, there is no difference in this effect between gouty and normal serum, and thus it seems unlikely that the serum effect influences which hyperuricaemic subjects develop urate crystal formation and hence gout.

We showed that gouty synovial fluid promotes urate crystal formation significantly more than does rheumatoid synovial fluid. That it was the gouty rather than the rheumatoid fluid which was abnormal was shown by the close similarity between the effects of CPPD and rheumatoid fluids. We further showed that the effect of gouty synovial fluid was not due to the presence of native urate crystals nor to an effect on the level of urate supersaturation. Our findings on the effect of gouty synovial fluid are in agreement with the previous small reports ${ }^{2} 36$ and suggest the possibility that patients who 
develop gout may have, in addition to hyperuricaemia, an abnormality of synovial fluid which promotes (or fails to inhibit) the formation of urate crystals. The failure of other crystal bearing fluids-for example, CPPD, to mimick that of gouty synovial fluid suggests that control mechanisms for biological crystal formation are specific for certain crystals. Similarly, CPPD and other non-urate crystals have been found not to promote urate crystal formation in vitro. ${ }^{7 \mathrm{a}} 14$

Investigation of the component of synovial fluid responsible for these effects is the basis of continuing studies in our department. Preliminary results indicate that the responsible component(s) is a macromolecule and sensitive to heat denaturation, characteristics which are compatible with a protein structure.

This work was supported by the Medical Foundation, University of Sydney. The authors would like to thank Dr Brigid Heywood, University of Bath, who performed the transmission electron microscopy and energy dispersive $x$ ray analysis.

1 Hall A P, Barry P E, Dawber T R, McNamara P M Epidemiology of gout and hyperuricaemia. A long-term Epidemiology of gout and hyperuricaemia.
population study. Am 7 Med $1967 ; 42: 27-37$.

2 Wilcox W R, Khalaf A A. Nucleation of monosodium urate crystals. Ann Rheum Dis 1975; 34: 332-9.

3 Tak H-K, Cooper S M, Wilcox W R. Studies on the nucleation of monosodium urate at $37^{\circ} \mathrm{C}$. Arthritis Rheum 1980; 23: $574-80$.

4 Burt H M, Dutt Y C. Growth of monosodium urate monohydrate crystals: effect of cartilage and synovial fluid components on in vitro growth rates. Ann Rheum Dis 1986; 45: $858-64$

5 Perl-Treves D, Addadi L. A structural approach to pathological crystallizations. Gout: the possible role of albumin in sodium urate crystallization. Proc $R$ Soc Lond [Biol] 1988; 235: 145-59.

6 Khalaf A A, Wilcox W R. Solubility and nucleation of monosodium urate in relation to gouty arthritis. Fournal of Crystal Growth 1973; 20: 227-32.

7 McGill N W, Dieppe P A. The effect of normal human serum on the growth of monosodium urate (MSU) crystals. $\mathrm{Br} \mathfrak{F}$ on the growth of monosodium ura
Rheumatol 1988; 27 (suppl 2): 93.

7a McGill N W, Dieppe P A. The effect of biological crystals and human serum on the rate of formation of crystals of monosodium urate monohydrate in vitro. $B r \mathcal{F} R$ heumatol (in press).

8 Arnett F C, Edworthy S M, Bloch D A, et al. The American Rheumatism Association 1987 revised criteria for the classification of rheumatoid arthritis. Arthritis Rheum 1988; 31: 315-24.

9 Katz W A, Schubert $M$. The interaction of monosodium urate with connective tissue components. $\mathcal{f}$ Clin Invest 1970; 49: 1783-9.

$10 \mathrm{Katz}$ W A. Role of proteoglycans in the development of gouty arthritis. In: Kelley W N, Weiner I M, eds. Handbook of experimental pharmacology. Vol 51. Uric acid. New York: Springer, 1978: 347-64.

11 Tak H-K, Wilcox W R, Cooper S M. Crystallization of monosodium urate and calcium urate at $37^{\circ} \mathrm{C} . \mathcal{F} \mathrm{Col}$ Interface Sci 1980; 77: 195-201.

12 Fiddis $R W$, Vlachos $N$, Calvert $P$ D. Studies of urate crystalisation in relation to gout. Ann Rheum Dis 1983;42 (suppl): 12-15.

13 Campion E W, Glynn R J, DeLabry L O. Asymptomatic hyperuricemia. Risks and consequences in the normative hyperuricemia. Risks and consequences

14 McGill N W, Dieppe P A. The specificity of promoters of monosodium urate (MSU) crystal formation. $B r \mathcal{J}$ Rheumatol 1989; 28 (suppl 1): 31. 